\title{
Joint Sampling-Communication Strategies for Smart-meters to Aggregator Link as Secondary Users
}

\author{
Mauricio C. Tomé, Pedro H. J. Nardelli, Hirley Alves, Matti Latva-aho \\ Department of Communications Engineering \\ University of Oulu \\ Oulu, Finland \\ Contact: nardelli@ee.oulu.fi
}

\begin{abstract}
This paper assesses the communication between smart-meters and aggregators as secondary users that transmit their data over the primary uplink channel. We assume: directional antennas are employed in the meter-aggregator link, secondary users transmit with limited power, meters' transmissions are randomized to avoid packet collisions, and an outage constraint for the secondary links' to guarantee their robustness. Therefore, the interference caused by the secondary users in the primary can be neglected. Conversely, the secondary users still experience interference from mobile users of the primary network, whose positions and traffic activity are unknown. Our goal is to study sampling-communication strategies for transmitting the average power demand of a household so the signal can be reconstructed by the aggregator with low deviation. Our results indicate that an event-based scheme based on energy packets can outperform the usual time-based, periodic, sampling.
\end{abstract}

Index Terms-Cognitive radio, sampling methods, smart grids, wireless networks

\section{INTRODUCTION}

One important key aspect when modernizing the power grids is the integration of communication networks including the final consumer [1]. For example, in the so-called smart grids, cloud computing applications [2] used for demand-side management and peer-to-peer energy trading would require efficient data processing and communication system.

Wireless systems offer a flexible and scalable option. While there exists the option of contracting the service from mobile operators, this would be expensive and might even saturate their network. Other alternative, which we will focus here, is to use the cognitive radio concept called spectrum sharing [3], where unlicensed - secondary - users want to transmit some information without disturbing the licensed - primary - users over the same frequency channel. One problem when deploying a system based on spectrum sharing is the complex decision-making dynamic among the secondary users [4].

This work is partly funded by Finnish Academy (Aka) and CNPq/Brazil (n.490235/2012-3) as part of the jointly funded project SUSTAIN, by Strategic Research Council/Aka BC-DC project (n.292854) and by the European Commission through the P2P-SmarTest project (n.646469).

978-1-4673-8463-6/16/\$31.00 (c) 2016 IEEE
If diverse applications and their respective requirements are taken into account, the cognitive radio solution becomes even more intricate and context-dependent [5]. In any case, the idea of spectrum sharing is already part of new generations of wireless systems [6]-[9]. Different smart grid applications, as presented in [10], are also planned to be deployed using cognitive radio. [11] presents an up-to-date survey on the topic.

This paper extends our recent results [12], where we studied a spectrum sharing scheme for the distribution grid scenario but only considering time-based sampling. There, we showed that such strategy creates redundant information allowing for higher outage probabilities with low root-mean-square deviation (RMSD) while optimizing the link throughput.

Here our focus is the trade-off between the sampling and communication strategies. We show the proposed event-based strategy requires in average less points for reconstructing the original signal. However, since there are less redundancy as well as points to be transmitted, the communication link must be much more reliable to reach a similar deviation when reconstructing the transmitted signal.

To carry out this analysis, we will use the database from [13], [14] to exemplify the average power demand curve. We deal with the uncertainty about the primary users by modeling them as a two-dimensional Poisson point process with a known density. In this way, we can obtain the outage probability for the communication link in closed-form and then optimize the link throughput subject to an outage constraint, as presented in [12]. By doing so, we can evaluate the impact of the outage probability in the RMSD when reconstructing the transmitted signal. The sampling strategy effects on the signal reconstruction are then studied for different outage probabilities, indicating the benefits of the event-based scheme.

The rest of this paper is divided as follows. Section II describes our system model focused on the spectrum sharing scheme. Section III presents the sampling methodologies used, namely time- and event-based. In Section IV, we provide the numerical results related to the proposed analysis. We conclude this paper in Section $\mathrm{V}$ providing discussions about our results and possible future works. 


\section{SYSTEM MODEL}

In this paper, we follow the system model introduced in [12]. Let us start by stating the assumptions of our model.

- Assumption 1: Spectrum-sharing scenario where licensed (primary) and unlicensed (secondary) users share the frequency bands allocated to the up-link channel.

- Assumption 2: Primary link is established between static cellular base-stations and mobile users. Secondary users are smart meters that send data to a given aggregator through the up-link channel, and their positions are fixed.

- Assumption 3: The smart meters transmit with limited power $W_{\mathrm{s}}$ such that $W_{\mathrm{s}} \leqslant W_{\max }$ where $W_{\max }$ is the maximum power allowed for the secondary users (which can be seen as an imposition from the primary network).

- Assumption 4: Smart meters associated with the same aggregator can perfectly coordinate their transmissions using time scheduling when time-based is used. When the event-based approach is used, the probability that a packet collision occurs tends to zero as far as their transmissions are naturally randomized.

Assumption 2 indicates that highly directional antennas in the secondary links can be worthy since the positions are fixed and orientation errors as defined in [15] can be avoided. Assumption 3 imposes the maximum range that the signal transmitted by the smart meters can reach. Therefore, radiation generated by the secondary link may be seen as a segment starting in the smart meter, passing through the aggregator and ending in a point related to $W_{\max }$.

The interference related to the proposed spectrum sharing can be then considered by looking at Assumptions 1,2 and 4: they indicate the co-channel interference occurs: (i) from smart meters to cellular base-stations, (ii) from smart meters to aggregators that they are not associated, and (iii) from mobile users to aggregators. From above, (i) and (ii) can be neglected when deploying either/both secondary or/and primary networks. Even when the locations are random, the chance of having a given terminal in the line segment approaches zero, reinforcing that such cases should not be considered.

Case (iii) is therefore the only one relevant and, to cope with it, we first need to model uncertainty of the mobile users' positions and traffic activity. We assume that the mobile users are distributed following a 2-dimensional Poisson point process $\Phi$ with density $\lambda$ in interferers per square-meter. The wireless channel is modeled by two components [16]: distance-dependent path-loss such that the received power decays with the distance and other related to fast-fading. The received power at the node of interest can be computed as $g_{i} r_{i}^{-\alpha}$, where $r_{i}$ is the distance between the reference receiver and the $i$ th node, $g_{i}$ is the channel gain between them, and $\alpha>2$ the path-loss exponent.

We focus our analysis on a reference smart meter-aggregator link, as shown in Fig. 1. During transmissions intervals, the interferers' positions and the channel gains do not change. The scenario is assumed interference-limited so the noise effects can be neglected without any qualitative difference [17].

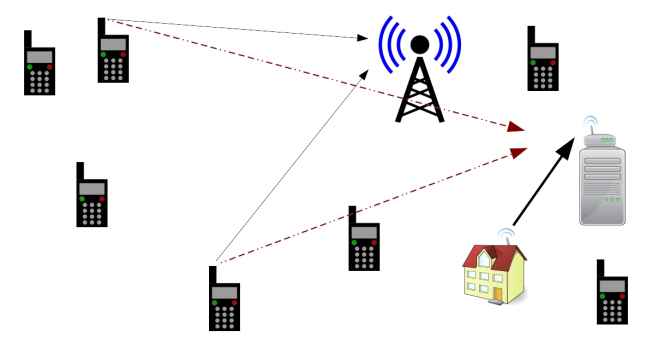

Fig. 1. An illustration of the proposed scenario, where primary and secondary users share the up-link channel. The reference smart meter (secondary transmitter) is depicted by the house, the aggregator (secondary receiver) by the CPU, the handsets are the mobile primary users (interferers to the aggregator) and the big antenna is the cellular base-station. As the smart meter uses directional antennas with limited transmit power (bold arrow), its interference towards the base-station can be ignored. The thin black arrows represent the primary users' desired signal, while the red ones represent their interference towards the aggregator.

If the primary users are equipped with omni-directional antennas and transmit with the same fixed power $W_{\mathrm{p}}$, the signal-to-interference ratio (SIR) at the aggregator can be computed as:

$$
\mathrm{SIR}_{0}=\frac{W_{\mathrm{s}} g_{0} r_{0}^{-\alpha}}{W_{\mathrm{p}} \sum_{i \in \Phi} g_{i} r_{i}^{-\alpha}}
$$

where the index 0 denotes the reference link.

The reference link employs both point-to-point Gaussian codes and the interference-as-noise decoding rule [18], [19], so that a spectral efficiency of $\log _{2}(1+\beta)$ in bits/s/Hz is achieved only if the SIR is greater than $\beta$. The probability $P_{\text {suc }}$ that a packet is successfully decoded by the aggregator is then the probability that $\operatorname{SIR}_{0}>\beta$; the outage probability in the communication is therefore $1-P_{\text {suc }}$.

To compute $P_{\text {suc }}$, we assume quasi-static channel gains (squared envelopes) $g$ that are independent and identically distributed exponential random variables (Rayleigh fading), and also a dynamic topology where interferers' positions change every transmission interval. Therefore, every transmission attempt can be viewed as a different realization of the point processes $\Phi$ and the channel gains $g$. We consider here that the distance between the reference meter and aggregator is known and has a fixed value $r_{0}=d$.

From these assumptions, we can find the highest throughput $T^{*}$ in bits/s/Hz that the secondary link can achieve subject to a maximum outage probability constraint $1-P_{\text {suc }} \leqslant \epsilon$ when the secondary transmit power $W_{\mathrm{s}}=W_{\max }$. Due to the space limitation, we do not present the details of the optimization procedure, which are found elsewhere [12]. The optimal throughput is then a function of the outage probability and given by:

$$
T^{*}=(1-\epsilon) \times \log _{2}\left(1+\frac{W_{\max }}{W_{\mathrm{p}}}\left(-\frac{\ln (1-\epsilon)}{\lambda \kappa \pi d^{2}}\right)^{\alpha / 2}\right) .
$$



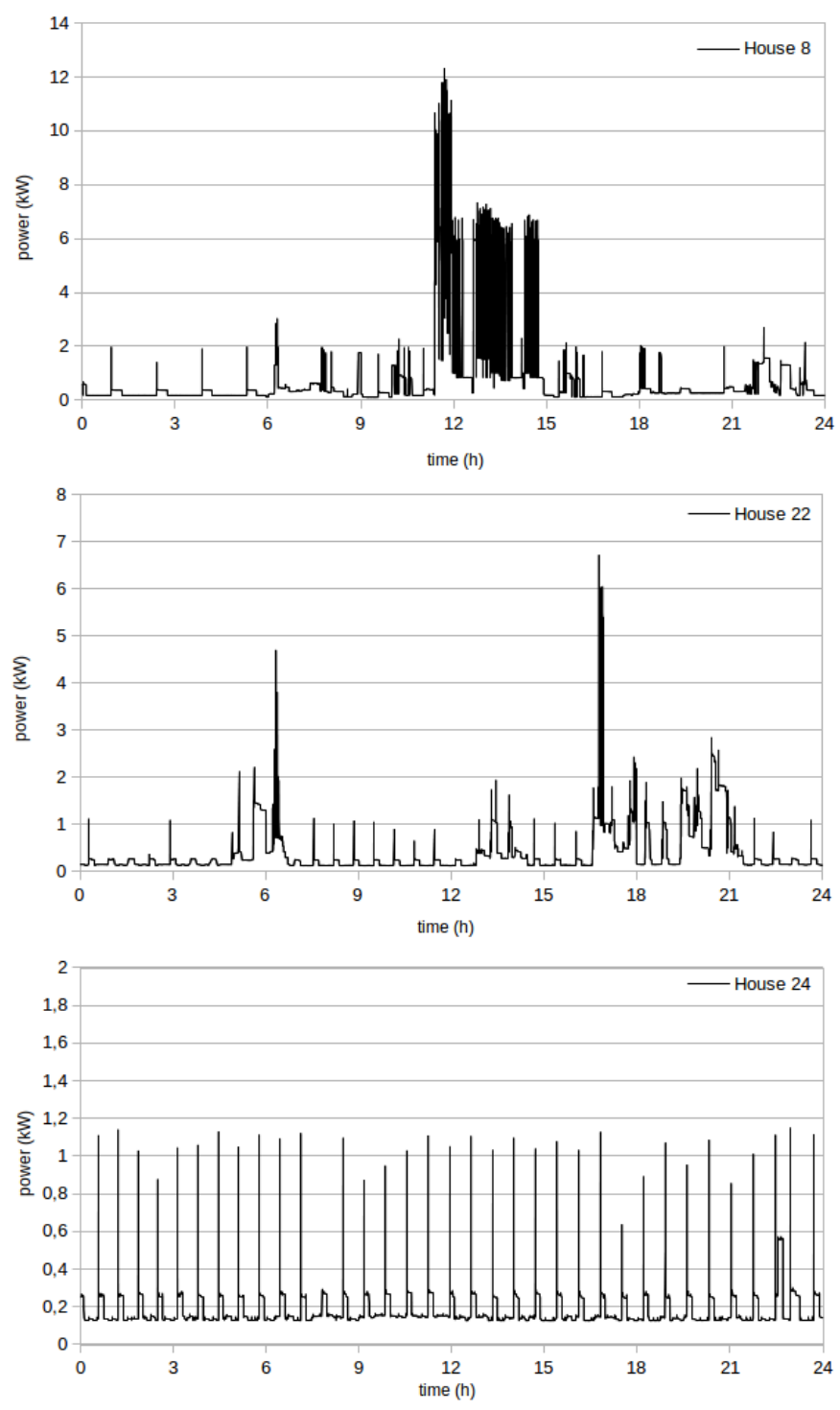

Fig. 2. Examples of the power readings from the REDD database (24 hour interval). Top: high power demand (house 8); Middle: intermediate demand (house 22); Bottom: Low power demand (house 24).

\section{SAMPLING METHODOLOGY}

\section{A. Simulation data}

The data used in the simulations comes from "The Reference Energy Disaggregation Data Set” (REDD) [13], [14]. The database consists of 6 houses where power demand was monitored over several weeks, with a resolution of seconds. The measurements from every house were divided into slices of 24 consecutive hours, and we discarded the slices that had missing data. This process rendered 53 slices of $24 \mathrm{~h}$, which can be seen as 53 individual households, and were used in the calculations of the time- and event-based measurements. Some examples of the resulting power demand over a 24-h timespan are shown on Fig. 2, and Fig. 3 shows the energy consumption from the houses.

\section{B. Measuring techniques}

1) Time-based sampling: The time-based sample is the most common sampling technique in use. For each one of the equally-spaced time intervals, $\Delta T$, the average power measurement is calculated, and associated to that time slice. ${ }^{1}$ This has some advantages as, for example, simplicity of the reconstruction of the average power consumption, and also disadvantages as privacy threat for the customer [20], [21].

In this paper, the simulated samples are taken every $\Delta T=$ 15 minutes, which is a common value used in smart metering deployment, which results in $n=96$ samples per day.

2) Event-based sampling: In the event-based approach, [22], the power consumption of the house is continuously monitored, but data is only sent when either: a) a certain amount of energy consumption $E$ is reached; or b) a sudden change in the power demand denoted by $\Delta P$ is detected. In this case, the information of interest is the energy consumption since the last packet was sent.

In this paper we set the thresholds: $E=0.5 \mathrm{kWh}$, and $\Delta P=0.5 \mathrm{~kW}$. These values are arbitrary and not optimal ${ }^{2}$; nevertheless, by using these values, we would like to verify whether a reduction of the number of transmitted points while guaranteeing low error when performing the data reconstruction is possible. We exemplify this in Fig. 4, where one can see that the chosen thresholds $E=0.5 \mathrm{kWh}$, and $\Delta P=0.5 \mathrm{~kW}$ result in a smaller number of points (on average) than when compared to $\Delta T=15$ minutes, with similar quality on the signal reconstruction.

To further reduce the amount of points sent, we avoid in our algorithm spikes in the power consumption, which are sudden variations on the power demand, such as the starting current of electric motors. Thus, herein, the spikes that lasts less than 2 seconds are discarded. An example of such events can easily be seen in the bottom part of Fig. 2 (house 24).

\section{Power demand reconstruction}

The power demand is reconstructed similarly in both cases. In the time-based strategy with 15 minute interval, we have

$$
P_{\text {avg,time }}[i]=\frac{E[i]-E[i-1]}{t[i]-t[i-1]}, i=\{1,2, \ldots, 96\},
$$

while, in the event-based strategy, we have

$$
P_{\text {avg,energy }}[j]=\frac{E[j]-E[j-1]}{t[j]-t[j-1]}, j=\{1,2, \ldots, n\},
$$

where $n$ is a value that we don't know beforehand. Even with $n$ unknown, the process of reconstructing the average power demand stays the same, with the slight difference that the granularity in the event-based strategy is in the scale of seconds, since a peak on $\Delta P$ or reaching a given $\Delta E$ threshold could

\footnotetext{
${ }^{1}$ In fact, to be able to compare both methods fairly, instead of sending the average power, we send cumulative energy values and the average power is reconstructed based on these values in a trivial manner. This method also work as a "fallback mode", since even if some of the packets are lost, it is still possible to keep track of the total energy consumption.

${ }^{2}$ Since each house has a different behavior and set of appliances, there's no single optimal value that will suit every house.
} 


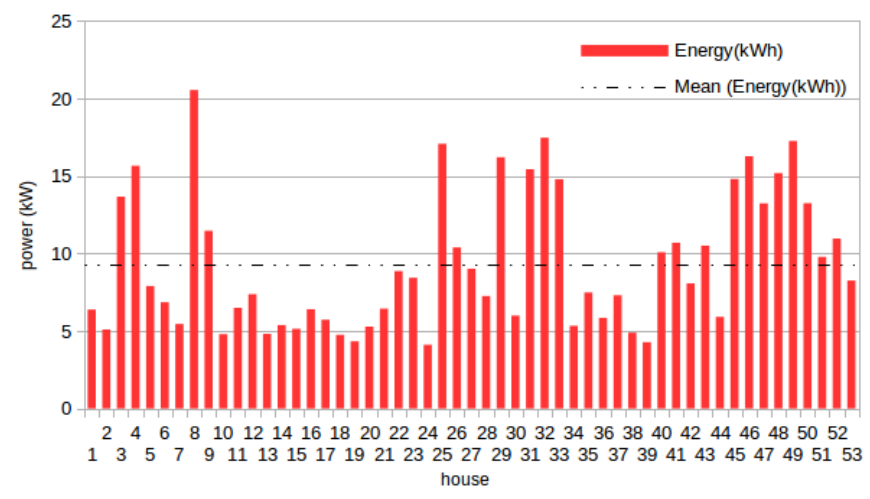

Fig. 3. Energy consumed per house during 24 hours and their mean value measured in $\mathrm{kWh}$

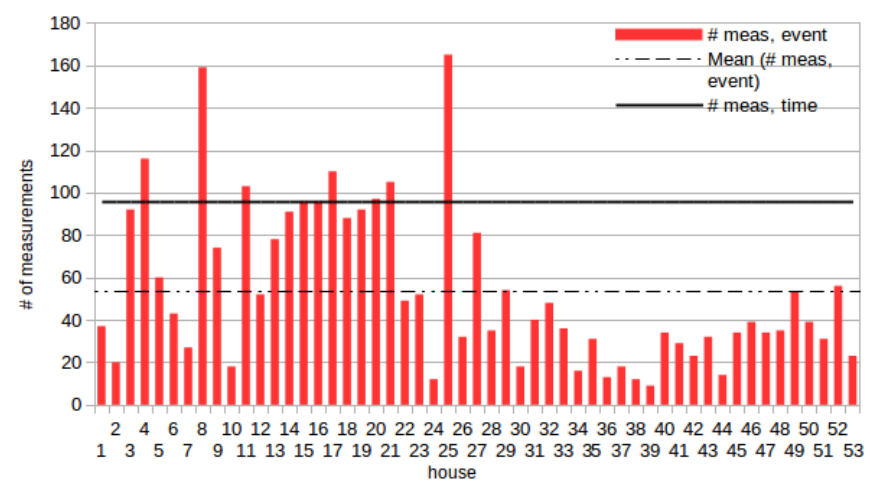

Fig. 4. Number of measures per house during 24 hours considering the timebased and the event-based scheme.
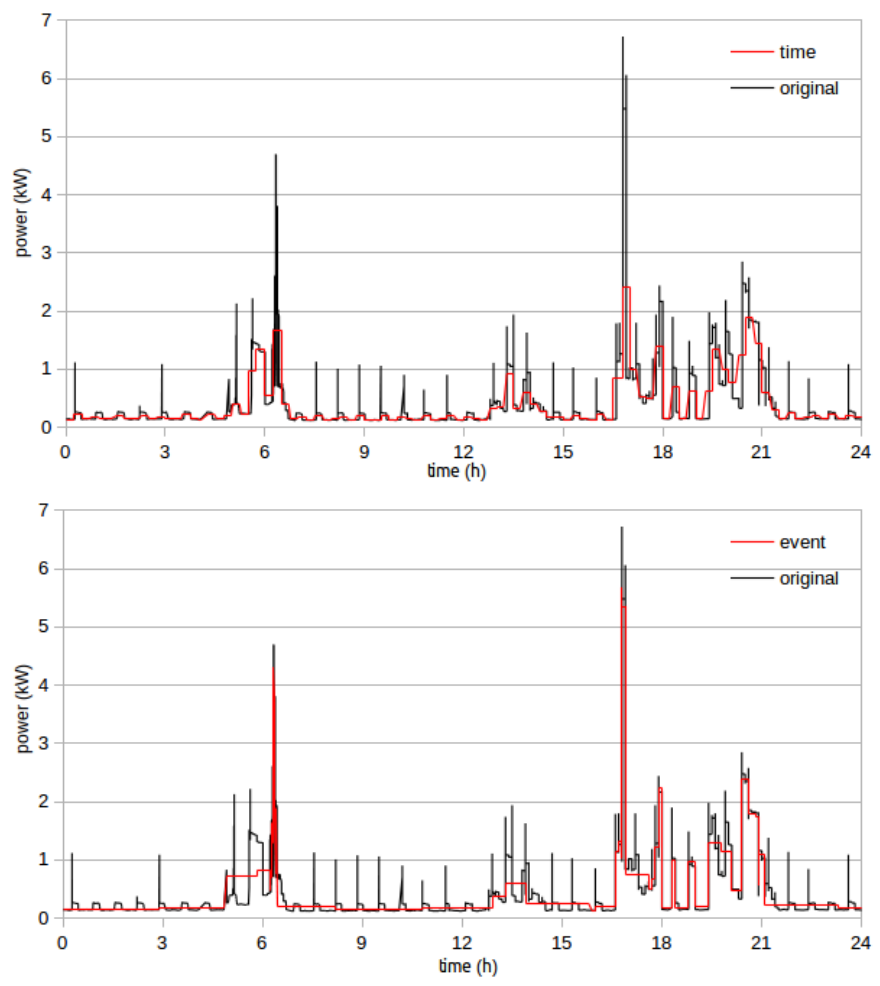

Fig. 5. Power demand reconstruction of house 22 during 24 hours, considering time-based (top) and event-based (bottom) measurements.

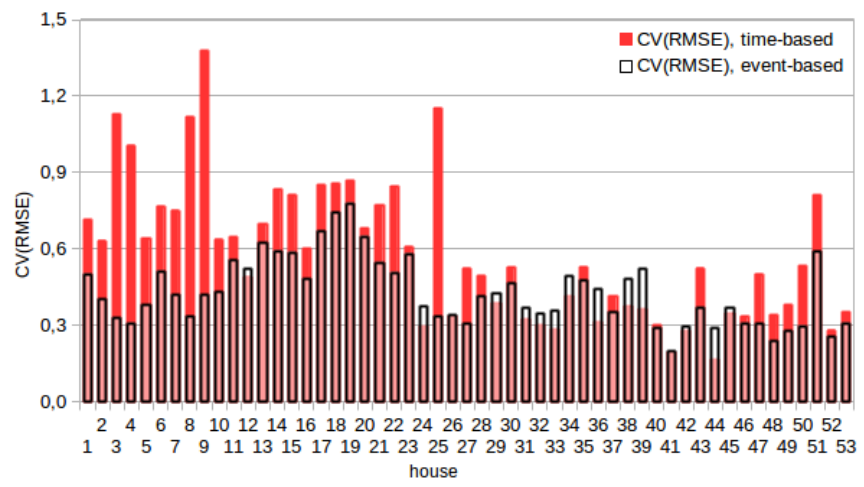

Fig. 6. Comparison between CV(RMSE) of time (red) and event (black outline) measures.

happen anytime. Fig. 5 shows the reconstruction of the power demand of house 22 using both methods.

\section{Estimation error}

In this case, we estimate the quality of the power demand curve estimation under the time-based and event-based strategies. We assume a perfect communication link so the outage probability is zero, which means that all the transmitted packages arrive at the destination.

To establish a comparison of the differences between the several measurements and the original data, the coefficient of variation of the root-mean-square deviation was used:

$$
C V(\mathrm{RMSD})=\frac{\mathrm{RMSD}}{\overline{P_{\text {orig }}}}=\frac{\sqrt{\sum_{t=1}^{n}\left(P_{\text {orig, }}-P_{\text {meas }, \mathrm{t}}\right)^{2}}}{\overline{P_{\text {orig }}}},
$$

where $P_{\text {orig,t }}$ is the set of original data for each house, $P_{\text {meas, }}$ is the set of averaged measures (either time- or event-based) and $\overline{P_{\text {orig }}}$ is the average power of the original data from each house. Fig. 6 shows the superimposed values of the error in reconstruction, for both sampling schemes. In most cases, we can see that even using an arbitrary value for the event-based thresholds it has a better estimation than the timed-based one.

\section{E. Estimation error, missing samples}

Following the case of reconstruction under a perfect reception scenario, we now analyze the case of signal reconstruction in presence of outages in the communication link with probability given by $\epsilon$, as in [12]. Remembering Section II, an outage event occurs due to co-channel interference from the primary users. As retransmissions of lost packets, an outage event implies that the transmitted sample is lost.

When the event-based strategy is considered, the data is transmitted in a randomized manner so the receiver has no knowledge about the missing information. To cope with that, every packet transmitted has cumulative information about the energy consumption. Therefore, even though the information about the average power demand is degraded, the information about the energy consumption is not lost. In this case, the power demand can be estimated during the communication outage event. We can then rewrite (4) as 

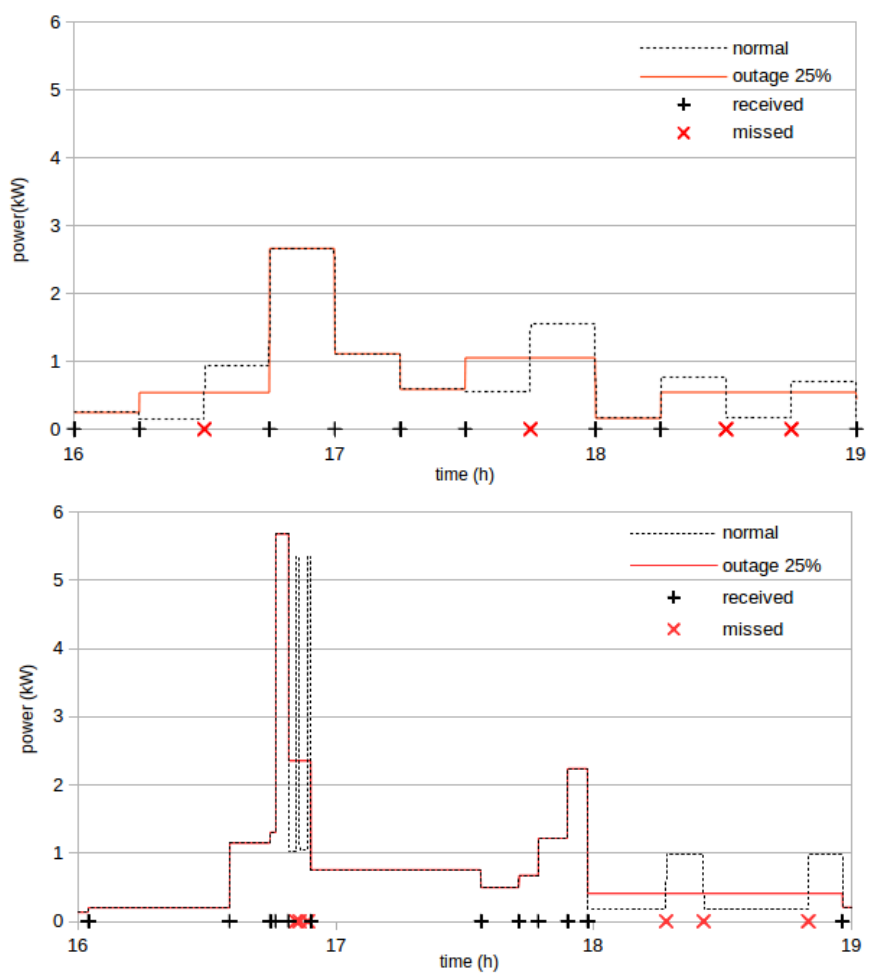

Fig. 7. Detail of the power estimation of house 22 under outage condition ( $25 \%$ of data loss), for the time-based (top) and event-based (bottom) strategy.

$$
\hat{P}_{\text {avg,energy }}[j]=\frac{\hat{E}[j]-\hat{E}[j-1]}{\hat{t}[j]-\hat{t}[j-1]}, j=\{1,2, \ldots, n\},
$$

where the hat ("^") symbol denotes that only the received samples are considered. Fig. 7 shows an example of how the power demand is estimated under outage conditions, and $C V$ (RMSD) for the outage conditions is calculated in a similar way as before, but now using $\hat{P}$ instead of $P$.

\section{RESUlTS}

Based on the data and concepts explained in the previous section, we now analyze the performance of the estimation under both strategies. We consider that the outage probability goes from 0 to $25 \%$, which are reasonable values for the communication network. Nevertheless, it is worth pointing out that $25 \%$ of outage probability may look too high value compared to standardized requirements for wireless metering, which required $98 \%$ of reliability [10]. As discussed in [12], such high value is a questionable choice as far as the signal reconstruction can be performed properly with, for example, $75 \%$ of reliability (i.e. outage constraint of $25 \%$ ).

From (2), increasing $\epsilon$ also increases the optimal link throughput for the system setting considered $\alpha=4, d=1$, $W_{\mathrm{p}}=1$ and $\lambda$ from 0.2 to $0.7, W_{\max }$ from 0.01 to 0.5 and $\epsilon$ from 0 to $25 \%$. Fig. 8 shows the effects of the outage probability $\epsilon$ on the reconstruction error which is reflected by the $C V$ (RMSD). Thus, we select the five representative households as follows:
- House 08: Biggest energy consumption;

- House 09: Best $C V$ (RMSD) value;

- House 22: Average power consumption;

- House 24: Smallest power consumption;

- House 44: Worst $C V$ (RMSD) value.

Note that even though the amount of houses was greatly reduced, the extreme cases - the biggest and smallest values of $C V$ (RMSD) - are covered and all the other cases lie in between. Since our objective is to assess the performance of the event-based estimation strategy in comparison of the timebased estimation, the errors shown in Fig. 8 are relative to each other; in other words, the graphic shows the difference between event-based estimation errors in comparison to timebased ones, in percentage: a negative error value indicates the event-based is better than the time-based, and vice-versa. It is possible to see in the results related to houses 8,9 and 22 that, even though the event-based estimation is still superior if compared to the time-based approach, the quality of the estimation degrades quicker.

On the other hand, for the houses 24 and 44, the error seems to be reduced, but we have to keep in mind that this is a relative comparison between the errors in time- and event-based estimation. In fact, the time-based estimation is degrading quicker than the event-based one. House 44 has similar power demand pattern as that of house number 24 , and, as such, the thresholds for the event-based measurement are set too high to make a proper representation of the power consumption. This explains both the low amount of measurements and the poor quality in the estimation. However, those cases do not pose a problem since the power demand is both low and almost constant.

This somehow atypical behavior is the power demand profile shown by house 24, which seems to be an empty house so power consumption is due to the stand-by appliances and refrigerator cycles. Nevertheless, those cases draw the attention to the fact that the limits for the event-based sampling strategy need to be carefully chosen to accurately represent the different households' consumption.

\section{CONCLUSION AND FUTURE WORK}

This paper presented an extension of [12] incorporating a joint analysis between sampling and communication system design. We compared here the more usual time-based, periodic, sampling against a event-based strategy using real data from electricity consumption. Our results showed that the event-based strategy provides an adequate way to represent the power demand data more accurately, leading to a lower number of samples (in average) to represent a load profile curve. In other words, this means less data to transmit and to store.

Our results further indicates that the event-based sampling can provide a comparable performance when communication errors exist. This means that it is possible to have an efficient sampling strategy, while the signal reconstruction is weakly affected by communication errors associated with primary 


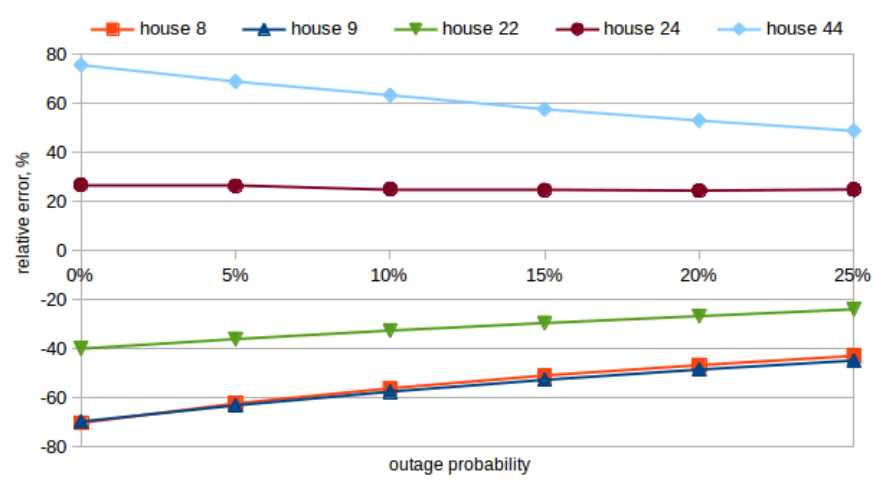

Fig. 8. Evolution of the relative error in the estimation, according to outage probability ( 0 to $25 \%$ ). Houses $8,9,22,24$ and 44 depicted

users in the proposed spectrum sharing technique. Furthermore, the strategy is simple to implement, and could be easily combined with any other demand as, for example, interleaved time samples, as shown in [23].

There are some cases, though, that the event-based representation is either poor (too little samples) or overrepresented (much more samples than the time-based strategy). To cope with them, a more theoretical description of the signal shall be provided looking the tools of compressed sensing and non-uniform sampling techniques [24], [25]. In this way, our future work in this topic will include strategies to dynamically optimize the event triggering, based on individual household consumption and appliances' usage patterns, so that all the houses will have a reduced number of samples without compromising their individual data.

\section{REFERENCES}

[1] P. H. J. Nardelli et al., "Models for the modern power grid," EPJ-ST, vol. 223, no. 12, pp. 2423-2437, 2014.

[2] S. Bera et al., "Cloud computing applications for smart grid: A survey," IEEE Trans. Parallel Distrib. Syst., vol. 26, no. 5, pp. 1477-1494, 2015.

[3] J. Peha, "Sharing spectrum through spectrum policy reform and cognitive radio," Proc. IEEE, vol. 97, no. 4, pp. 708-719, Apr. 2009.

[4] B. Wang et al., "Game theory for cognitive radio networks: An overview," Computer Netw., vol. 54, no. 14, pp. 2537-2561, 2010.

[5] A. Ahmad et al., "A survey on radio resource allocation in cognitive radio sensor networks," Commun. Surveys Tuts., vol. 17, no. 2, pp. 888917,2015

[6] C. H. de Lima et al., "Coordination mechanisms for self-organizing femtocells in two-tier coexistence scenarios," IEEE Trans. Wireless Commun., vol. 11, no. 6, pp. 2212-2223, 2012.

[7] L. D. Xu et al., "Internet of things in industries: A survey," IEEE Trans. Ind. Informat., vol. 10, no. 4, pp. 2233-2243, Nov. 2014.

[8] S. D. T. Kelly et al., "Towards the implementation of IoT for environmental condition monitoring in homes," IEEE Sensors J., vol. 13, no. 10, pp. 3846-3853, Oct. 2013.

[9] A. Bradai et al., "EMCOS: Energy-efficient mechanism for multimedia streaming over cognitive radio sensor networks," Pervasive Mob. Comput., vol. 22, pp. 16-32, 2015.

[10] M. Kuzlu et al., "Communication network requirements for major smart grid applications in HAN, NAN and WAN," Computer Netw., vol. 67, pp. 74-88, 2014.

[11] A. A. Khan et al., "Cognitive radio for smart grids: Survey of architectures, spectrum sensing mechanisms, and networking protocols," Commun. Surveys Tuts., 2015, to appear.

[12] P. H. J. Nardelli et al., "Maximizing the link throughput between smartmeters and aggregators as secondary users under power and outage constraints," Ad Hoc Netw., 2015, in press. Arxiv ID: 1506.04830.
[13] J. Z. Kolter and M. J. Johnson. [Online]. Available: http://redd.csail.mit.edu/

[14] -REDD: A public data set for energy disaggregation research," in Workshop on Data Mining Applications in Sustainability (SIGKDD), San Diego, CA, vol. 25 . Citeseer, 2011, pp. 59-62.

[15] J. Wildman et al., "On the joint impact of beamwidth and orientation error on throughput in directional wireless Poisson networks," IEEE Trans. Wireless Commun, vol. 13, no. 12, pp. 7072-7085, Dec. 2014.

[16] M. Haenggi, Stochastic geometry for wireless networks. Cambridge University Press, 2012.

[17] S. Weber et al., "An overview of the transmission capacity of wireless networks," IEEE Trans. Commun., vol. 58, no. 12, pp. 3593-3604, Dec. 2010.

[18] F. Baccelli et al., "Interference networks with point-to-point codes," IEEE Trans. Inf. Theory, vol. 57, no. 5, pp. 2582-2596, May 2011.

[19] P. H. J. Nardelli et al., "Throughput analysis of cognitive wireless networks with Poisson distributed nodes based on location information," Ad Hoc Netw., 2015.

[20] G. Eibl and D. Engel, "Influence of data granularity on smart meter privacy," IEEE Trans. Smart Grid, vol. 6, no. 2, pp. 930-939, 2015.

[21] H. Alves et al., "Enhanced transmit antenna selection scheme for secure throughput maximization without CSI at the transmitter and its applications on smart grids," IEEE Trans. Inf. Forensics and Security, Nov 2015, submitted. Arxiv ID: 1511.06518.

[22] M. Simonov, "Event-driven communication in smart grid," IEEE Commun. Lett., vol. 17, no. 6, pp. 1061-1064, 2013.

[23] M. Simonov et al., "Gathering process data in low-voltage systems by enhanced event-driven metering," IEEE Syst. J, vol. PP, no. 99, pp. 1-12, 2015.

[24] D. L. Donoho, "Compressed sensing," IEEE Trans. Inf. Theory, vol. 52, no. 4, pp. 1289-1306, Apr. 2006.

[25] M. Miskowicz, "Send-on-delta concept: An event-based data reporting strategy," Sensors, vol. 6, no. 1, pp. 49-63, 2006. 